\title{
Psoriasiform keratosis: a psoriatic lesion in a non psoriatic patient
}

\author{
Solé Juan Javier, Luna Paula Carolina*, Larralde Margarita and Casas José Gabriel \\ Alemán Hospital, Buenos Aires, Argentina
}

\section{Introduction}

Psoriasiform Keratosis is a seldom reported entity, characterized by a single, scaly plaque mainly located on the extremities with histological features of psoriasis in a patient with no history of psoriasis. The etiopathogenesis remains unknown.

We here-by report the case of a 64-year-old man presenting a solitary and asymptomatic scaly plaque, located on the right arm, with no response to topical corticosteroids. A punch biopsy was performed and histology was consistent with psoriasiform keratosis.

\section{Case report}

A 64 year old otherwise healthy man consulted for an asymptomatic, solitary, well-defined, scaly plaque of $1.5 \mathrm{~cm}$ located on the right arm, of two weeks of duration (Figure1). No other lesions were observed elsewhere. He was treated with clobetasol for one month. Given the lack of response to topical steroids a biopsy was performed.

Histopathology showed parakeratosis, intracorneal neutrophils, regular acanthosis and dilated capillaries in the papillary dermis with a mild perivascular infiltration in the upper dermis consistent with psoriasis (Figures 2 and 3).

Given the patient lacked other cutaneous or ungual lesions and the

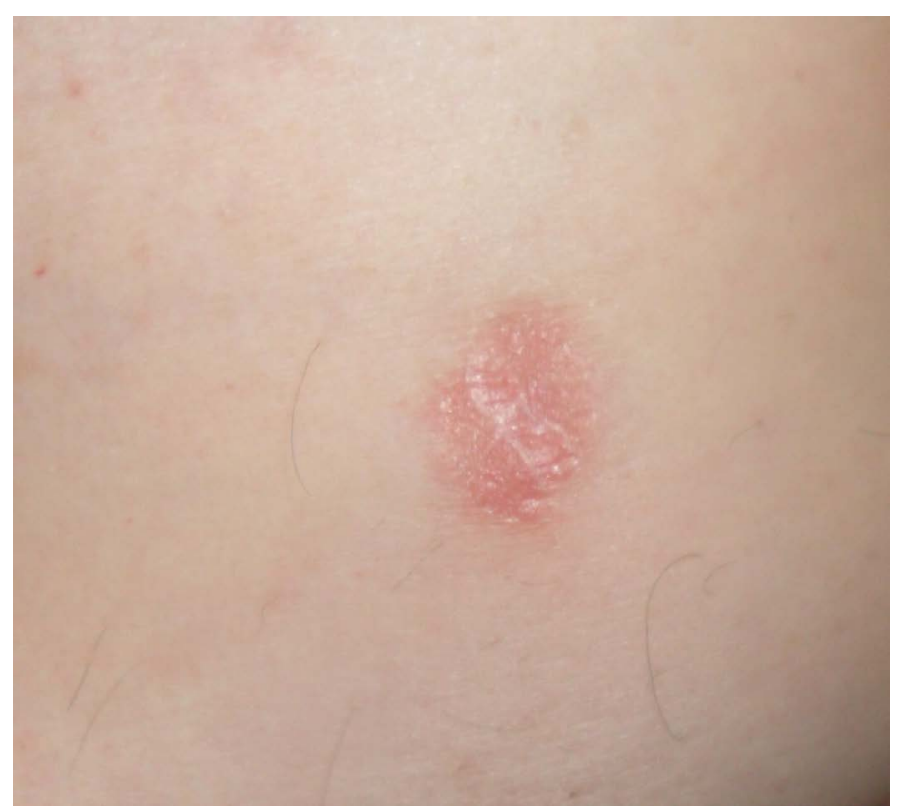

Figure 1. Solitary well defined scaly plaque on the inner arm.

showed no response to topical steroids the diagnosis was consistent with Psoriasiform keratosis.

\section{Discussion}

The term Psoriasiform keratosis (PK) was coined by Walsh et al. [1] to describe a lesion characterized by a solitary, well-defined, scaly papule or plaque, with histopathologic features closely resembling psoriasis in patients with no other psoriatic lesions at the time of diagnosis or follow-up [2].

A literature review reports only five previous publications of this entity, describing 34 lesions [1-5].

Of the few reported cases, both sexes seem to be similarly affected. It tends to affect mainly the elderly, although one lesion has been described in a 25 year old patient [3]. Most lesions have been described as erythematous, scaly to crusted papules and plaques, mainly over the limbs, although any part of the skin might be affected. The known duration of lesions ranged from 1 week to 2 years [1].

Histopathologic findings as originally described by Walsh et al. [1] resemble that seen in psoriasis and includes regular or sometimes irregular acanthosis, with focal to confluent mounds of parakeratosis intercalated with collection of neutrophils throughout the cornified

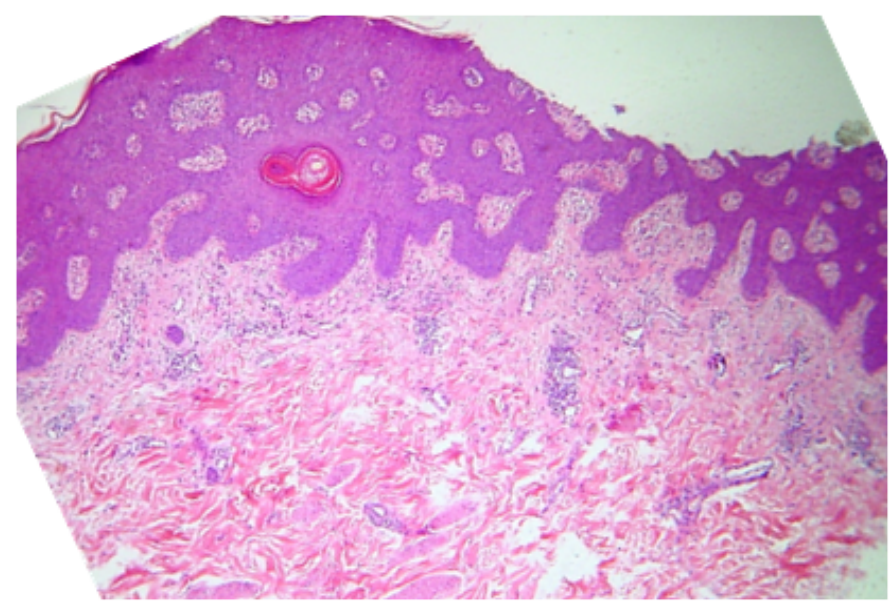

Figure 2. Regular acanthosis, dilateted capillaries.

Correspondence to: Luna Paula Carolina, Alemán Hospital, Buenos Aires, Argentina, E-mail: Paulacarolinaluna@gmail.com

Received: March 03, 2016; Accepted: March 28, 2016; Published: March 31, 2016 


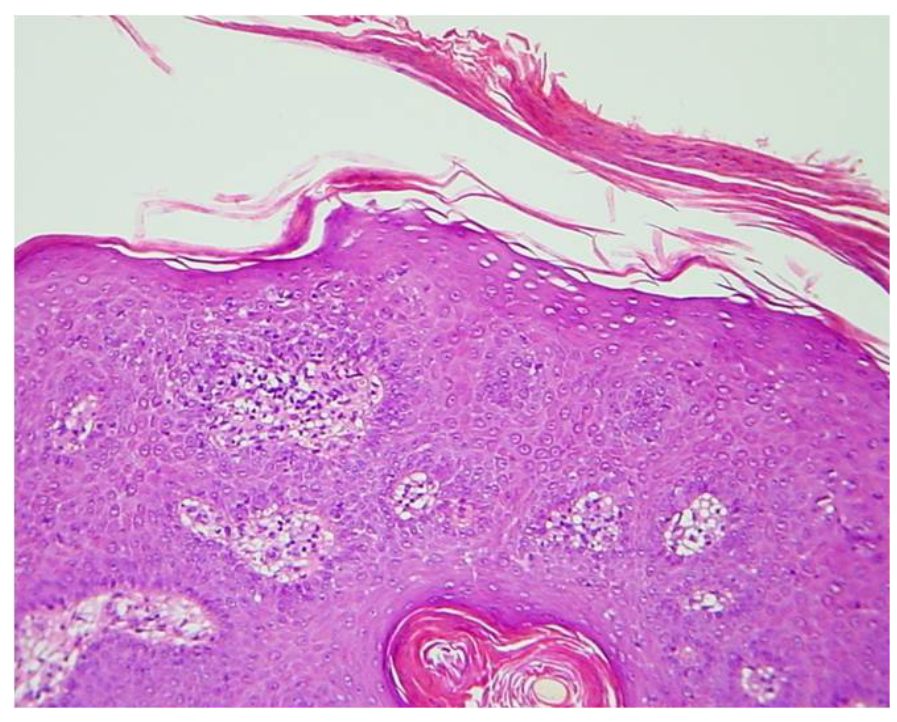

Figure 3. Parakerathosis

layer together with intraepidermal spongiform pustules. Ectatic small vessels in the papillary dermis and a sparse superficial perivascular lymphocytic infiltrate similar to that seen in psoriasis vulgaris have also been reported [2].

The clinical course of the lesions and the lack of response to topical steroids suggest that they probably correspond to an epithelial proliferation with the histological features of psoriasis but with no true relation to this disease [3]. This "phenomenon", where a peculiar inflammatory reaction pattern typically seen in widespread diseases appears as solitary lesions, has been previously described for other skin diseases: Porokeratoma (solitary tumor-like Mibelli's porokeratosis), Epidermolityc acanthoma (Solitary or few lesions with histological picture of bullous congenital icthyiosiform erythroderma) and the lichenoid keratosis (single lesion liken planus-like) [3].
Sezer et al. demonstrated the presence of papilloma virus (HPV) type 6-specific DNA via direct sequencing of a sample of one patient with PK. Although this type of HPV has been described in other tumors and acanthomas, further studies will be needed to determine if HPV type 6 is an etiological factor in the pathogenesis of this disorder or has been just an incidental finding [2].

Clinical differential diagnosis includes seborrheic keratosis, Bowen disease, squamous cell carcinoma, basal cell carcinoma, clear cell acanthoma, and verruca vulgaris [2].

Lesions don't show a tendency towards spontaneous regression. Reported treatments with topical steroids, as in our patients, and other topical treatments have shown not to be effective. This is why physical treatments such as simple surgical excision should be considered [3].

The etiopathogenesis is unknown and some authors believe that PK should be classified in the group of cutaneous acanthomas [4].

In conclusion, the diagnosis of PK should be taken into account when approaching a solitary erythematous patch with the histological features of psoriasis but does not resolve with topical steroids and develops in non-psoriatic patients [3].

\section{References}

1. Walsh SN, Hurt MA, Santa Cruz DJ (2007) Psoriasiform keratosis. Am J Dermatopathol 29: 137-140. [Crossref]

2. Sezer E, Böer A, Falk T (2010) Identification of human papilloma virus type 6 in psoriasiform keratosis. Am J Dermatopathol 32: 492-494. [Crossref]

3. Carbone A, De Simone C, Valenzano F, Amerio P, Massi G (2009) Psoriasiform keratosis. Eur J Dermatol 19: 631-632. [Crossref]

4. Mutasim DF (2007) Psoriasiform keratosis: a lesion mimicking psoriasis. Am J Dermatopathol 29: 482-484. [Crossref]

5. Pires CA, Sousa BA, Nascimento Cdo S, Moutinho AT, Miranda MF, et al. (2014) Psoriasiform keratosis - case report. An Bras Dermatol 89: 318-319. [Crossref]

Copyright: (C2016 Javier SJ. This is an open-access article distributed under the terms of the Creative Commons Attribution License, which permits unrestricted use, distribution, and reproduction in any medium, provided the original author and source are credited. 